vollen Betriebe, so liebt der Unterdruck, wolchen der Motor in der Saugleitung erzeugt, den ganzen Körper $E$ so hoch, daß die bei $J$ zuströmende Frischluft auBen vorbeistreichen kann. Der Brennstoff steigt dabei in dem Rohre $R$ empor, da auf den Brennstoffspiegel im Suhwimmerbehälter der AuBenluftdruck wirkt, und mischt sich in der erforderlichen Weise mit der Frischluft. Beim Ankurbeln und bei Leerlauf des Motors geht hingegen auBen an der Kappe $E$ keine Luft vorbei, da der geringe Unterdruck nicht genügt, um diesen Teil von dem Gehäuseteil $F$ abzuheben. Dagegen streicht die Luft durch die viel engeren Kanäle $T$ und $V$ und erzeugt an der oberen Mündung des Rohres $R$ einen solchen Unterdruck, daß die Vergasung auch bei niedriger Umdrehungszahl aufrechterhalten bleibt. Da sich bei tiefster Stellung der Kappe $E$ auch der freie DurchfluBquerschnitt an der Nadel $P$ verkleinert, so kann in den Düsenraum $G$ nur cine beschränkte Brennstoffmenge nachfließen. Der

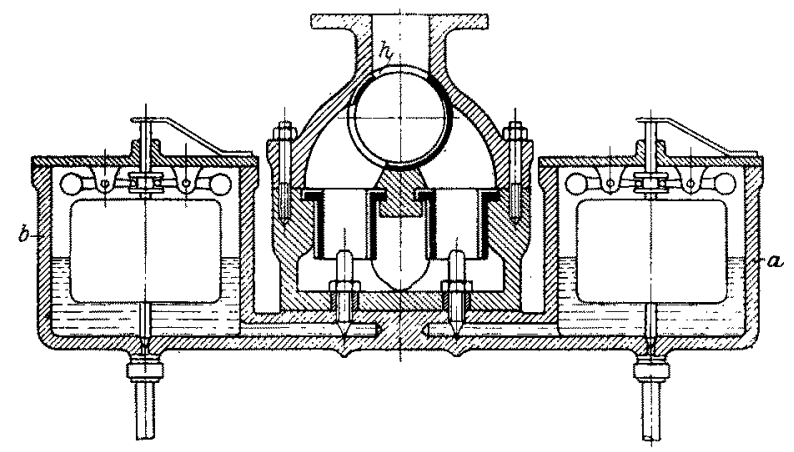

Wiz. S. Hoppelvergaser.

Mantel $B$ wird an den Kühler oder die Auspuffleitung angeschlossen und dient dazu, das fertige Gemisch zu erwärmen und die Verdampfung des Brennstoffes $z \mathbf{u}$ beschleunigen.

Die Verbesserungen, die man bei den Vergasern angebracht hat, um mit Rücksicht auf den hohen Preis von leicht verdampfbarem Benzin auch schwerer flüchtige Rohöldestillate und insbesondere Benzol für den Betrieb von Motorwagen benutzen zu können, beschränken sich im wesentlichen auf die Heizung ähnlich wie bei dem vorstehend beschriebenen Vergaser sowie auf Anderungen in Luftzuführung, entsprechend dem höheren Luftgehalt, den das fertige Gemisch haben muB. Auch hier wird der Fortschritt äuBerst mühsam erkämpft. da man systematische Versuche der hohen Kosten wegen scheut und sich auf das Ausprobieren von Neukonstruktionen auf dem Versuchsstand besehränkt. Da jeder Automobilmotor, wenn er einmal warm geworden ist, sogar auch mit Petroleum ganz gut läuft, so könnte man insbesondere für Lastwagen- und Bootsmotoren recht gut die sogenannten Doppelvergaser anwenden, die sich in früheren Jahren, als man noch ernsthaft an die Zukunft des Spiritusbetriebes glaubte, gut bewährt haben. Ein solcher Doppelvergaser besteht nach Fig. 8 aus zwei getrennten Vergasern, deren Schwimmergehäuse $a$ und $b$ an Behälter mit Benzin und Petroleum angeschlossen sind und deren spritzdüsen je nach der Einstellung des Schiebers $h$ abwechselnd zur Wirkung gelangen. Beim Ankurbeln und beim Leerlauf läBt man die Benzindüse arbeiten, während der Hauptzeit der Fahrt dagegen kann die Petroleum- oder Benzoldüse benutzt werden. Der Verbrauch an Benzin wird hierbei so eingeschränkt, daß der Benzinpreis kaum eine Rolle spielt.

Das immer notwendiger werdende Bestreben. dem Automobilbetrieb billigere, schwerer verdampfende Brennstoffe dienstbar zu machen, lenkt unwillkürlich unsere Aufmerksamkeit auf die Frage, welche Zukunft wohl unseren heutigen Vergasern bevorstehen mag. Obgleich derartige Ausblicke in die Zukunft wegen der sprunghaften Entwicklung unserer Technik immer etwas Gewagtes an sich haben, kann man doch schon heute sagen, dab das allgemeine Streben dáraufhin abzielt, den heutigen Vergaser grundsätzlich aufzugeben und das ganze Arbeitsverfahren des Automobilmotors abzuändern. Wenn es gelingt, Vorrichtungen zu finden, die es gestatten, den Brennstoff unmittelbar in den $\mathrm{Mo}^{-}$ torzylinder einzuspritzen, so sind nicht allein alle heutigen Vergaserschwierigkeiten beseitigt, weil die Verdampfung dann im Innern des heißen Zylinders erfolgt und das Mischungsverhältnis durch genaues Zumessen der eingespritzten Brennstoffmenge bestimmt werden kann, sondern man ist auch in der Lage, Brennstoffe zu verwenden, die außerhalb des Motorzylinders überhaupt nicht verdampft werden kömen, z. B. sehwere Teeröle usw. Vorläufig ist man von diesem Ziele allerdings noch sehr weit entfernt, denn gewöhnliche Kolbenpumpen u. dergl. sind zur Einführung der außerordentlich kleinen. Brennstoffmengen, die bei Antomobilmotoren in Frage kommen, nicht geeignet.

\section{Die 23. Jahresversammlung der Deutschen Zoologischen Gesellschaft.}

\author{
Von Hanns v. Lengerken, Berlin
}

Der diesjahrige Kongres der deutschen Zoologen in Bremen wurde durch eine Ansprache von Professor Korschelt eröffnet. Professor Schauinsland begrüBte die Versammlung im Namen des Bürgermeisters und der Stadt Bremen und gab zugleich einen kurzen AbriB der Entwicklungsgeschichte des Städtischen Museums und der naturwissenschaftlichen Bestrebungen in der freien Reichs und Hansestadt. Nach Erledigung des geschäftlichen Teils folgte ein umfangreiches Referat Professor Meisenheimers uber: AuBere Geschleohtsmerkmale und Gesamtorganismus in ihren gegonseitigen Beziehungen. Das Referat möge bier schon des allgemeineren Interesses wegen etwas ausführlicher besprochen werden.

Der Vortragende beginat bei den Protisten. Aus den ursprünglich hier vorhandenen Isogameten werden Anisogameten, Mikro- und Makrogameten. Bei Coccidien triit uns schon eine dimorphe Ausbildung der Mutterzellen entgegen. Es sind hier schon zwei Generationen in den Dienst der Geschlechtsfunktion getreten, die Ga. meten nebst Mutterzellen und die Gametocyten. Die Agen lassen bereits drei Generationen unterscheiden, 
nimlich die Gameten selbst, die Gametocyten und den Gametocytenträger. Diese drei Generationen bilden die Grundlage der geschlechtlichen Differenzierung aller niederen Pflanzen und ebenso fast sämtlicher Metazoen. - Es folgt eine Betrachtung des Dimorphismus, wie er bei den Prothallien der Farne häufig ist, und eine Beleuchtung der entsprechenden Verhältnisse bei höheren Pflanzen. Darn wird die Entstehung der Getrenntgeschlechtlichkeit aus der Zwitterorganisation besprochen, und zwar bei Bilhurzia und Wedlia. Höhere Pflanzen, Gametocytenträger 2. Ordnung, weisen Vorgänge sexueller Differenzierung aut, die mit denen bei Trematoden und Ciliaten in Parallele zu stellen sind. - Der weiteren Betrachtung unterliegt der Gametocytenträger, der bei allen Algen, Pilzen, bei geschlechtlichen Gene. rationen der Moose und Farne, wie bei fast allen Tieren vorhanden ist. Dieser Gametocytenträger ist zunächst geschlechtlich indifferent. Erst bei höher differenzierten Metazoen begegnen wir einer geschlechtlichen Umbildung des Gametocytenträgers, und es treten die sekundären Ge schlechtsmerkmale auf. Es ist selbstverständlich, daß die Geschlechtscharaktere immer an das Vorhandensein vestimmter Geschlechtsdrusen gebunden sind. Bei Hermaphroditen können die Sexualcharaktere zu Artmerkmalen werden; bei getrenntgeschlechtichen Tieren bildet sich durch den Einfluß der Geschlechtsdrüsen der geschlechtliche Dimorphismus aus, der sich sogar bis zu Verschiedenheiten der Hämolymphe und der Darmzellen durchbilden kamn. Die Kastratiou hat im allgemeinen einen Verlust der Sexualcharaktere zur Folge. - Es finden sich jedoeh auch Sehmetterlingszwitter, die äußerlich genau halbiert sind, innerlich aber nur weibliche Keimdrïsen zeigen. Sehr jung kastrierte Raupen beiderlei Geschlechts lieferten Falter, die einen der ursprünglichen Geschlechtsdrüse entsprechenden Geschlechtshabitus aufwiesen. Sehr interessante Resultate haben bekanntlich die Gonadentransplantationen geliefert. steinacher verpflanzte Ovarien auf Männchen von Ratten und Meerschweinchen. Es zeigte sich, daß die Geschlechtsmerkmale auf der jeweiligen Entwicklungsstufe stehen blieben, dagegen wurden die Brustwarzen weiblich, und es zeigten sich typisch weibliche Charaktere und weibliche Psyche. Die Transplantation von Geschlechtsorganen bei Schmetterlingen hat keinerlei Einfluß auf die entgegengesetzten sekundären Gieschlechtsmerkmale. Es werden die bekannten Fälle, die den spezifisehen EinfluB dex Geschlechtssubstanz auf die Ausbildung äuberer Sexualmerkmale bezeugen, besprochen. Die Brücke zwischen den sich widersprechenden Tatsachen findet Meisenheimer in der Auffassung, daß, je juinger, phylogenetisch gedacht, das Geschlechtsmerkmal ist, es um so abhängiger von der Fegenwart einer Sexualdrüse sein wird. Je alter, je fixierter das Merkmal ist, desto weniger wird es von der Abwesenheit der Geschlechtsdrüse beeinflußt werden. Im wesentlichen dienen also zwei Momente zur Aufklärung der Beziehungen zwischen Keimdrüse und sekundiren Geschlechtsmerkmalen, und zwar sind das Stoffwechselzustände und Verschiedenartigkeit phyletischer Entwicklungsstufen.

Der Vortragende geht dann auf die Vererbungserscheinungen der Geschlechtscharaktere ein. Es tritt bald intermedillre Vererbung, bald Mosaikvererbung auf, in anderen Fitlen sind Mendelsche Spaltungsregeln zu beobachten. Es ist anzunehmen, daB in einem Individuum die Determinanten des entgegengesetzten Geschlechts latent vor handen sind. Es wären sonst Vererbungen von Eigenschaften des Vaters mitterlicher Seite durch die Mutter wuf ihren Sohn unerklärlich. Hat erst einmal der erworbene Geschlechtscharakter beim Männchen seinen maximalen Positivwert erreicht, so beginnt er auch, sich auf das Weibchen zu ibertragen; so daß zuletzt der Dimorphismus der sekundiren Geschlechtsmerkmale in bezug auf bestimmte Organe fortfallen k:inn.

In der zweiten Sitzung trug A. Thienemann über Die Balzwassertierwelt Westfalens vor.

Der Relerent konnte in westfälischen Salzwissern 120 Tierarten nachweinen. Mehr als die Haltte davon besteht aus Dipteren und Coleopteren, der Rest rekrutiert sich aus Gastropoden, Nematoden, Cladoceren, Trichopteren und Protozoen. Es fehlen gänzlich z. B. Hydroiden, Hirudineen, Amphibien usw. Es finden sich Tiere, die auch anterswo vorkommen, es sind dies die "Custe" (haloxene Formen). Salzwasserliebhaber (halophile Formen) sind Organismen, die auch im SüBwasser gedeihen, die aber doch eine Vorliebe für Salzwasser zeigen. Die eigentlichen typischen Salztiere (Halobien) leben fast nur in Salzwasser. Sehr interessant ist die ainBerst schnelle Besiedelung von Salzwässern mit Tieren. Der Einfluß des Salagehaltes auf die Zusammensetzung der Fauua ist sehr groß. Bei $22 \%$ Salzgehalt wird das Wasser azoisch.

Es folgte Spengels Vortrag Zur orgrnisation und Sistematik der Gattung Sipumalus.

AuBer einigen fur die systematik der Sipunculiden wichtigen Untersuchungen wird ein am Hinterende von Sipuneulus gelegenes Sinnesorgan, wahrscheinlich eiu Statocyste, beschrieben.

Nachdem in der 3. Sitzung der nächstjahrige Vorstand gewählt und über die Abanderung der Statuten abgestimmt war, sprach $k$. Escherich über: Dor gegenwärtige Stand der angewandten Entomologic und Vor sehläge zu deren Verbesserung.

Wie absolut zeitgemäß die vielfachen Anregungen und Forderungen Escherichs waren, beweist die sich dem Vortrag anschlieBende und sehr lebhafte Diskussion. Escherioh stell die Vernachlässigung der angewandten Entomologie in Deutschland sehr überzengend dar. Amerika muB, was die Förderung der volkswirtschaftlich so wichtigen Probleme der praktischen Entomologie anbetrifft, durehaus vorbildlich sein. Es hat eine genügende Zahl von Arbeitsplätzen errichtet, beschaftigt ein großes Personal in seinen Instituten und hat an seinen Universitäten sehr gute Ausbildungsstätten für griindlich zu schulende Entomologen. Wie steht es dagegen in Deutschland? Die wenigen Institute, an denen praktische entomologische Fragen gelöst werden, sind schnell aufgezählt. Sie reichen lange nicht aus, um den Berg ungelöster Fragen zu erledigen, Fragen, die für Forst- und Landwirtschaft im Heimatlande und in den Kolonien von größter Wichtigkeit sind. Wir können in Deutschlant demjenigen Zoologen, der sich der angewandten Entomologie widmen will, noch nicht einmal die erforderliche Ausbildung verschaffen, wenn wir uns eben nicht ans Ausland wenden wollen. Daher ist die Gründung entomologischer Professuren ein dringendes Bedürfnis. AuBerdem fehlt uns eine Zentralanstalt in bezug auf die Verteilung der Arbeitsgebiete usw. Um nun diese Ubelstände, denen aus volkswirtschaftlichen Gründen allein schon abgeholfen werden müßte, schneller zu beseitigen, hat sich die Deutsche Gesellschaft für angewandte Wntomologie unter dem Vorstand von Professor Escherich, Professor Behuangart, Professor Heumons und Dr. Martini gebildet, deren Bestrebungen von jedem Finsichtigen durchaus unterstuitzt werden sollten.

I'. van Bemmelen behandelte Die phylogenetische Bedeutung der Puppenseichnung bei den Rhopaloceren und ihre Beziehungen zu derjenigen der Rawpen und Imagines. tan Bemmelen hat die Puppen von Pieriden, Papilioniden und Nymphaliden untersucht und kommt zu dem 
Schluß, daß nicht nur auf den Flügelscheiden, sondern auch auf dem ganzen übrigen Körper eine Farbenzeichnung auftritt, der eine phylogenetische Bedeutung beizumessen ist. Die farbigen Lepidopterenpuppen geben wahrscheinlich ein Bild der Farbenzeichnung bei den Ahnen der Tagschmetterlinge. Die einfarbigen Puppen haben sicherlich infolge verborgener Lebensweise die ursprüngliche Farbe verloren.

Fr. Voß referierte über Vergleichende Untersuchungert über die Flugwerkzeuge der Insekten.

Der Vortragende hat verschiedene Flugeinrichtungen bei Insekten eingehend untersucht und drei Grundtypen der Flugorgane aufgestellt. Die verschiedenen Typen und Modelle in aerodynamischer Beziehung zu klären, würde zur Verbesserung der menschlichen Flugzeuge, vielleicht sogar zur Nachbildung tierischer Flugapparate führen.

In der 4. Sitzung lieb sich 71 . Lohmam uber Coccolithophoriden aus.

Diese gerade in der Flachsee so massenhaft aultretenden Flagellaten unterwirft der Referent einer eiugehenden Untersuchung in bezug au ihre Systematik. Nach der Ausbildung des Skelettes zerfällt die Familie in zwei naturliche Unterfamilien; die eine bant ihre Schalen aus undurchbohrten, einfach scheibenförmigen Discolithen, die andere aus durchbohrten Trehmalithen auf. Die letzteren bestehen aus einem Röhrenstüek mit sich nach dem Rande zu verjüngender Kalkscheibe. Die Geißelzahl schwankt zwischen eins und zwei.

Paul Schulze sprach dann uber Chitin und andes. Cuticularstrukturen bei Insekten.

Die sehr interessanten Untersuchungen beginnen mit der Erklärung des Banes der Käferflägeldecken im allgemeinen. Es werden zwei Typen unterschieden. Der eine Typ weist zwei Platten auff, die durch kleine Säulen (columnae) verbunden sind (Melasoma). An der ventralen sitzen kleine Dornen (spinulae) oder Chitinperlen. (perlae). Im Hohlraum zwischen den beiden Platten liegt nun das von Panl $S c h_{u} z_{\approx e}$ entdeckte Karotingewebe, oder er ist durch sekundäres Chitin ganz ansgefullt. Beim zweiten Typ tritt als neuer Bestandteil der Decke cine braune Schicht zwischen der Grenzlamelle und der Chitinhauptlage anf, die „Lackschicht". Die Säulen, Bestigungselemente nach dem Prinzip der T-Trüger, bilden sich bei Typus 1 schon während des Puppenstadiums; bei Typus 2 entstehen sie erst nach dem Schlüpfen des Käfers. Der zentrale Teil der Säulen be. steht beim 2. Typ ganz aus Lacksehichtsubstanz. Es werden dann die verschiedenen Schichten der Deck" eingehend geschildert. Als Resultat ergibt sich, da $B$ die Tackschicht kein eigentliches Chitin ist. Sie weist typisch sechseckige Felderung, der Form der Bildungszellen entsprechend, auf. Das Chitin selbst besteht aus Platten mit fibrillärem Bau. In den Aussparungen befindet sich die nicht fibrilliire Zwischensubstanz. Paul Schulze bespricht ferner das Sekretrelief bei Cicindelen und einigen Coleoptera. Bei Cicindela tritt eine Reliefskulptur aus nicht ganz regelmäBigen Sechs. ecken auf. Von besonderer Wichtigkeit sind zwei Elemente der Skulptur: die schuppenförmigen Erhebungen (Cyrthome) und die Kurvenanfangspunkte, von denen die Sechsecke in kreisspiraliger Anordnung ausgehen. Da sich die Reliefskulptur in Kalilauge löst, bestelit sie nicht aus Chitin. Außerdem hat die Sekretschicht aucl noch für das Zustundekommen der EIytrenfärbung Bedeutung.

In der 5. Sitzung diente Der Flug der Tiere H. Erhard zum Vortragsthema.

Beim Tierflug kommt nur das Prinzip der Aerodynamik in. Betracht. Der Flug vieler Tiere ist ein "Fallschirmflug"s (Flattermaki, Draco). Den typischen "Gleitflugs" findet man bei den Flugfischen. Den primi- tivsten „Ruderflug“, den ,Flatterflug“, zeigen die Fledermäuse. Er besteht aus einem Ausbreiten und Zusammen. falten der Flugel. Die Insekten bedienen sich eines je nach der Spezies sehr modifizierten Flatterfluges; der Hinterleib dient dabei als Steuer. Bei Käfern mit harten Elytren spielen beim Flug die Alae die Hauptrolle. Meister des Flnges sind die Vögel. Gute Flieger besitzen lange, schmale, geringe Krümmung aufweisende Flügel schlechte Flieger kurze, breite, stark gekrümmte Flügel. Beim spiraligen Schwebeflug ohne Flügelschlag spielen vertikale Luftströmungen eine große Rolle. Der Ruder flug ist am sehwierigsten zu erklären. Um vorwärts zu kommen, schlägt der Vogel von oben hinten nach unten vorn. Beim Niederschlagen erfolgt eine Hebung und Beschleunigung, beim Aufschlagen eine Verzögerung. Der Hauptmoment des Vogelfluges ist die automatisehe Stabilisierung. Bei der Möve z. B. spielt auch noch die Stellung des Schwanzsegels eine große Rolle; so ist der Schwanz beim Kreisen sehr weit auseinandergebreitet. Ganz kleine Vogel bedienen sich fast stets des Wellenfluges. Unsere menschlichen Flugwerkzeuge sind iuber hundertmal so ungünstig im Gesamtnutzeffekt gestellt als die Vögel im Durchschnitt.

Es folgten mehrere kürzere Referate, auf die einzeln einzugehen der Raum nicht gestattet. E. Breßlau sprach Ober das spezifische Gewicht des Protoplasmas und die Wimperleraft der Turbellarien und Infusorien; E. Martini Ober die Stellung der Nematoden in System; $G$. Whts jun. brachte eine vorläufige Mitteilung: Cytologische Beobachtungen an Polytoma wella, und Prell demonstrierte Deutsohe Proturen.

Als Ort der nächstjährigen Versammlung wurde Freiburg gewählt.

\section{Über die Entwicklung von Lichtsinn und Farbensinn in der Tierreihe ${ }^{1}$ ).}

Von Geh. Hofrat Prof. Dr. C. v. Hep. München.

v. Hep hat im Laufe der letzten Jahre eino Reihe neuer Methoden zur Untersuchung des Farbensinnes der Tiere entwickelt und berichtet in seinem Vortrage an der Hand zahlreicher Projektionsbilder von Blitzlichtaufnahmen verschiedener Tierarten im Spektrum über die Ergebnisse seiner Untersuchungen, in erster Linie solcher mit spektralen Lichtern. Wir müssen uns hier auf die Wiedergabe einiger weniger seiner Befunde beschränken.

\section{Wirbeltiere.}

Dex Affe sieht das Spektrum am langwelligen und am kurzwelligen Ende genau so weit wie wir. auch die adaptativen Änderungen bei längerem Dunkelaufenthalte und Herabsetzung der Lichtstärke der Reizlichter sind dort die gleichen wis bei uns. Tagvögel und Reptilien sehen das Spektrum am langwelligen, roten Ende eben so weit wie wir, dagegen ist es am kurzwelligen, violetten Ende für sie hochgradig verkürzt, nämlich bis zur Gegend des Blaugrün, d. h. diese Sauropsiden können das Grünblau, Blau und Violett des Spelstrums nieht mehr wahrnehmen; es hat dies seinen Grund in dem Vorhandensein rot und gelb gefärbter, sogenannter Ölkugeln vor den lichtempfindlichen Elementen der

1) Autoreferat des Vortrages auf der 85. Vorsammlung Dentscher Naturforscher und Arzte in Wien, September'1913. 